\title{
Metabolomic Profiles of Men and Women Ischemic Stroke Patients
}

\author{
Nicolas Poupore ${ }^{1}\left(\mathbb{D}\right.$, Renee Chosed ${ }^{1}\left(\mathbb{D}\right.$, Sergio Arce ${ }^{1}$, Robert Rainer ${ }^{2}$, Richard L. Goodwin ${ }^{1}$ \\ and Thomas I. Nathaniel ${ }^{1, *(1)}$
}

1 School of Medicine Greenville, University of South Carolina, Greenville, SC 29605, USA; npoupore@email.sc.edu (N.P.); chosed@greenvillemed.sc.edu (R.C.); sarceuss@gmail.com (S.A.); GOODWIRL@greenvillemed.sc.edu (R.L.G.)

2 PRISM Health Upstate, Greenville, SC 29605, USA; Robert.Rainer@prismahealth.org

* Correspondence: nathanit@greenvillemed.sc.edu; Tel.: +1-8644559846; Fax: +1-8644558404

check for updates

Citation: Poupore, N.; Chosed, R.; Arce, S.; Rainer, R.; Goodwin, R.L.; Nathaniel, T.I. Metabolomic Profiles of Men and Women Ischemic Stroke Patients. Diagnostics 2021, 11, 1786. https://doi.org/10.3390/

diagnostics 11101786

Academic Editor: Edoardo Gaude

Received: 21 August 2021

Accepted: 22 September 2021

Published: 28 September 2021

Publisher's Note: MDPI stays neutral with regard to jurisdictional claims in published maps and institutional affiliations.

Copyright: (C) 2021 by the authors. Licensee MDPI, Basel, Switzerland. This article is an open access article distributed under the terms and conditions of the Creative Commons Attribution (CC BY) license (https:// creativecommons.org/licenses/by/ $4.0 /)$.

\begin{abstract}
Background: Stroke is known to affect both men and women; however, incidence and outcomes differ between them. Therefore, the discovery of novel, sex-specific, blood-based biomarkers for acute ischemic stroke (AIS) patients has the potential to enhance the understanding of the etiology of this deadly disease in the content of sex. The objective of this study was to identify serum metabolites associated with male and female AIS patients. Methods: Metabolites were measured with the use of untargeted, reverse-phase ultra-performance liquid chromatography-tandem mass spectrometry quantification from blood specimens collected from AIS patients. Samples were collected from 36 patients comprising each of 18 men and women with matched controls. Metabolic pathway analysis and principal component analysis (PCA) was used to differentiate metabolite profiles for male and female AIS patients from the control, while logistic regression was used to determine differences in metabolites between male and female AIS patients. Results: In female AIS patients, 14 distinct altered metabolic pathways and 49 corresponding metabolites were identified, while 39 metabolites and 5 metabolic pathways were identified in male patients. Metabolites that are predictive of ischemic stroke in female patients were 1-(1-enyl-palmitoyl)-2-arachidonoyl-GPC (P-16:0/20:4) $(\mathrm{AUC}=0.914,0.765-1.000), 1$-(1-enyl-palmitoyl)-2-palmitoyl-GPC (P-16:0/16:0) (AUC = 0.840, 0.6561.000), and 5,6-dihydrouracil (P-16:0/20:2) (AUC $=0.815,0.601-1.000)$. Significant metabolites that were predictive of stroke in male patients were 5alpha-androstan-3alpha,17beta-diol disulfate (AUC = 0.951, 0.857-1.000), alpha-hydroxyisocaproate (AUC $=0.938,0.832-1.000$ ), threonate (AUC $=0.877,0.716-$ 1.000 ), and bilirubin (AUC $=0.817,0.746-1.000)$. Conclusions: In the current study, the untargeted serum metabolomics platform identified multiple pathways and metabolites associated with male and female AIS patients. Further research is necessary to characterize how these metabolites are associated with the pathophysiology in male and female AIS patients.
\end{abstract}

Keywords: ischemic stroke; metabolomics; male patients; female patients

\section{Introduction}

Stroke presents a more significant health burden on women than in men as women present with more stroke events and are less likely to recover [1]. While age-specific stroke rates are higher in men, women present with more stroke incidents than men because of their longer life expectancy and much of a higher incidence at older ages [2]. Similar to age-adjusted mortality, women present with more overall lower age-adjusted stroke incidence than men [3,4]. In addition, functional outcomes and quality of life after stroke are consistently poorer in women, and women are reported to present with worse prestroke disability than men [3,5]. Moreover, men and women with stroke differ concerning the prevalence of stroke risk factors [6,7]. Women with stroke are older at onset and are more likely to have atrial fibrillation and hypertension [8]. In contrast, men with stroke are more likely to present with a history of heart disease, myocardial infarction, 
peripheral arterial disease, diabetes, and alcohol and tobacco use $[9,10]$. The most common biological explanation for differences in stroke between men and women is posited to be hormonal [11]. In addition, studies in animal models demonstrate that women have smaller stroke volumes than men [12].

The human blood contains a wide variety of chemically diverse low molecular weight compounds, the metabolome, which can be measured in parallel through modern metabolomic technologies [13]. Analysis of the metabolome in AIS patients provides new opportunities to understand the pathophysiology of ischemic stroke [14]. Therefore, metabolomics is a promising technique for the evaluation of global metabolic changes in stroke [13]. By comparing metabolic profiles and their dynamic changes, changes in pretreated and treated patients can be elucidated. Metabolites in blood have been reported to be associated with vascular diseases [15]. For example, lipoprotein associated phospholipase A2 (Lp-PLA2) was reported to be linked with atherosclerosis and transient ischemic attack [16,17], and lysophosphatidylcholines (LysoPCs) were significantly associated with recurrent stroke [18]. In addition, metabolic profiles in AIS are reported to be significantly different from healthy people $[19,20]$. For example, changes in sphingomyelin and phosphatidylcholine metabolism were independently linked with risk of infarction in healthy adults. While different, there are multiple blood-based biomarkers investigated in AIS patients. For example, multiple blood biomarkers (N-terminal probrain natriuretic peptide [NT-proBNP], d-dimer, S100 $\beta$, neuron-specific enolase, vitamin D, cortisol, interleukin-6, insulin, uric acid, and albumin) were effective in the identification of patients with increased possibility of cardioembolism and AF [21]. Moreover, various blood-based biomarkers including BNP/NT-proBNP, d-dimer, CRP, TNF- $\alpha$, IL-6, and IL-1 are reported to be significantly associated with ischemic stroke [21-23]. Findings from the existing studies indicate that metabolomics is a powerful tool that can be used to explore biomarkers and related pathways in stroke.

The vulnerability to several diseases and the response to treatments differ between men and women [24]. Therefore, the differential treatment for men and women AIS patients would represent personalized medicine approach [25] to provide care for AIS patients. However, this requires an extensive understanding not only clinical risk factors but also the intrinsic molecular differences between men and women AIS patients. As a basis for a gender-specific care for AIS patients, the characterization of the molecular differences between the men and women AIS patients is necessary. Moreover, affected pathways may reveal genderspecific susceptibility. Knowledge of the underlying metabolic differences might lead to concrete starting points for a future research to improve care for men and women AIS patients. Existing studies [13,14,26-34] that investigated metabolites in stroke focused on differences between stroke and control, hemorrhagic strokes compared with non-hemorrhagic or ischemic strokes. For example, elevated plasma DNA concentrations were detected in patients with hemorrhagic strokes compared with non-hemorrhagic strokes, with a $31 \%$ sensitivity and $83 \%$ specificity for discriminating the two types of stroke [34]. Moreover, plasma levels of miR-124-3p, miR-125b-5p, and miR-192-5p, were found to be elevated and correlated positively with infarct volume of stroke patients [35-37]. In addition, protein biomarkers for brain injury and thrombosis were categorized and used to discriminate hemorrhagic stroke from ischemic stroke patients [38]. A recent study by Daokun et al. [13] analyzed serum metabolites and risk of ischemic stroke in men and women patients. The results reveal circulating biomarkers for stroke and novel pathways for AIS and its subtypes. However, differences between men and women patients were not reported. While findings from existing studies $[13,14,26-34]$ highlight the potential of metabolomics for discovering novel circulating biomarkers for stroke and its subtypes, existing studies did not report genderdifferentiated results. Therefore, the specific metabolites and related pathways that are directly associated with men and women AIS patients are not fully understood. Therefore, we conducted an untargeted metabolomics study to investigate whether metabolic profiles and related pathways are different in men and women patients with AIS. 


\section{Methods}

Approval for this study was obtained from the PRISMA Health Institutional review board (Pro00072801), and all methods were performed in accordance with the relevant guidelines and regulations. A total of 36 ischemic stroke patients comprising each of 18 female and male ischemic stroke subjects with matched controls were recruited for this study. Healthy controls were randomly selected from PRISMA Health system. Samples were collected from patients within $24 \mathrm{~h}$ of symptom onset based on relevant ischemic lesions on CT or brain MRI. The general criteria for inclusion were as follows: at least 18 years of age and meeting the diagnostic criteria for ischemic stroke. Patients with cardiac, kidney, or liver failure, acquired immunodeficiency syndrome, inflammatory bowel disease and systemic infection were excluded. Morning, fasting blood samples were collected from 36 ischemic stroke patients comprising each of 18 female and male ischemic stroke subjects with matched controls. The processing and preparation of samples have been described in a previous study [39]. Briefly, samples were subjected to methanol extraction and then divided into aliquots for analysis by ultrahigh performance liquid chromatography/mass spectrometry (UHPLC/MS). The global biochemical profiling analysis was comprised of four unique arms. These were reverse phase chromatography positive ionization methods optimized for hydrophilic compounds (LC/MS Pos Polar) and hydrophobic compounds (LC/MS Pos Lipid), reverse phase chromatography with negative ionization conditions (LC/MS Neg), as well as a HILIC chromatography method coupled to negative (LC/MS Polar) [40]. These methods alternated between full-scan MS and data-dependent MSn scans. The scan range covered $70-1000 \mathrm{~m} / \mathrm{z}$ with slight variation. Automated comparison of the ion features in the experimental samples to a reference library of chemical standard entries that included retention time, molecular weight $(\mathrm{m} / \mathrm{z})$, preferred adducts, and insource fragments as well as associated MS spectra and curated by visual inspection for quality control using software developed at Metabolon was used to identify metabolites. Identification of known chemical entities was based on comparison to metabolomic library entries of purified standards [41].

\section{Data Analysis}

Data reduction was performed using phase and baseline correction. The corrected spectra, which correspond to the chemical shift, were imported into AMIX 3.9.5 (Bruker Biospin, Rheinstetten, Germany); all spectra were reduced into integral regions with equal lengths. Regions that contained the resonance from residual water were set to zero. To reduce the concentration differences between samples, data were normalized to the total spectral area, and datasets were analyzed by pattern recognition methods using the software packages (Simca-P, version 11.5 (UmetricsAB, Umea, Sweden), and MetaboAnalyst 3.0 (www.metaboanalyst.ca, accessed on 15 September 2021). Skewed distributions for more symmetric distribution were determined by transforming the data to nonlinear conversions, while maximum variation between samples was determined using a PCA decomposition approach. This was used to determine whether the samples could be differentiated based on overarching components representing each sample's global metabolite profile. Individual metabolites that did not have any variation between the samples were removed from the PCA analysis. The data were analyzed using a direct oblimin rotation to obtain a non-orthogonal solution. After plotting samples onto a graph based on their two components, outliers were removed from the PCA based on if they visually skewed the data. The PCA was performed for both female and male patients separately. The specific metabolites between classes were interpreted using variable importance in projection (VIP) and the correlation coefficient. A pairwise comparison was performed between the male and female stroke patients to discover distinct biomarkers associated with male and female patients among the thousands of variables using Student T-tests. The level of significance was set at $p<0.05$.

To select biomarker candidates for men and women patients, we used a logistic regression model. The data was split into training and validation, and the backward logistic 
regression model was built on the training sample set to determine the best metabolite combination. After calculating multivariate analysis, the specific metabolites between classes were selected using VIP. Metabolites with VIP scores $>1.0$ in the partial least squares (PLS) were examined and selected for their discrimination power by multiple statistical criteria. VIP values were used as one method of identifying biomarkers with predictive value in separating men from women. Metabolites with VIP scores $\leq 1$ at each time point were considered irrelevant to the prediction and excluded from analysis. A similar approach was used by other studies $[25,28]$. The predictive ability of the model for each time point was internally validated based on leave-one-out cross validation using the Q2 diagnostic statistic. In addition, logistic regression and ROC analysis (calculated from the logistic regression) were used to establish a diagnostic model of metabolites for men and women patients. The ROC allowed us to identify metabolites that may represent candidate biomarkers for male and female AIS. The area under the ROC curve (AUC) value was used as a measure of the prognostic accuracy of individual metabolites. Therefore, while ROC curve analyses were performed for the designed model, the performance of each biomarker model was assessed using the AUC to determine sensitivity and specificity.

All variables were adjusted for in our analysis, and the effect of confounding variables did not mask our results. Just as any typical clinical studies, AIS patients who were admitted for treatment vary widely, and some related factors may confound the results of our analysis. In this study, we considered the following potentially confounding variables: gender is potentially a confounder, since the stroke incidence differs between men and women, including hospitalization rates [42]. Age at admission by admission date misused birth date as a confounder was categorized according to a related study [43]. Because comorbidities and complications associated with medication or infections were associated with AIS [20,21], and could mask the interpretation or our metabolomics results, we considered medications used including use of antibiotics, anti-hypertensive, use of cholesterol reducing medication medications, antiplatelet medications. Comorbidities' including chronic kidney disease, atrial fibrillation disease, carotid artery stenosis, diabetes and systemic infection were considered as confounders. Treatments including the use of tissue plasminogen activator (rtPA), thrombectomy or mechanical removal of clots were added as confounding variables in our analysis. Other confounders considered in our analysis were disease status at admission and discharge including mild, severe and critical based on NIHSS stroke severity evaluation.

The metabolite profiles and pathways were analyzed for both men and women patients to reveal the metabolic network reprogramming of AIS with detailed impact using metabolomic pathways analysis of the MetaboAnalyst 3.0 software. This allowed us to determine both pathway enrichment and pathway topology, which identifies the most relevant metabolic pathways that are differentially affected in male and female ischemic stroke patients.

\section{Results}

A total of 1322 biochemicals, 1062 named compounds of known identity, and 260 unnamed compounds of unknown structural identity were identified. Of this, 55 metabolite levels were significantly different between the ischemic and control group in the female cohort (Table 1). The PCA did not reveal distinct potential biomarkers between the ischemic and control groups for the female population (Figure 1). As shown in the figure, component 1 encompassed $21.399 \%$ of the variance, and component 2 encompassed $10.783 \%$ of the data. The biological pathway analysis for female patients revealed fourteen different metabolic pathways (Figure 2 and Table 2), including glycerophospholipid metabolism, pantothenate, and CoA biosynthesis, beta-alanine metabolism, linoleic acid metabolism, pyrimidine metabolism, alpha-Linolenic acid metabolism, glycerolipid metabolism, seleno compound metabolism, alanine, aspartate and glutamate metabolism, phosphatidylinositol signaling system, arachidonic acid metabolism, biosynthesis of unsaturated fatty acids, tryptophan metabolism and aminoacyl-tRNA biosynthesis. 
Table 1. Metabolite differences between ischemic stroke patients and control patients in the female population.

\begin{tabular}{|c|c|c|c|c|c|}
\hline Biochemical & Super Pathway & Sub Pathway & Control & Ischemic & $p$-Value \\
\hline Alanine & Amino Acid & $\begin{array}{c}\text { Alanine and Aspartate } \\
\text { Metabolism }\end{array}$ & $1.29 \pm 0.3$ & $1.04 \pm 0.16$ & 0.043 \\
\hline Indoleacetate & Amino Acid & Tryptophan Metabolism & $1.62 \pm 1$ & $0.82 \pm 0.39$ & 0.039 \\
\hline Isovalerate (C5) & Amino Acid & $\begin{array}{l}\text { Leucine, Isoleucine and } \\
\text { Valine Metabolism }\end{array}$ & $2.11 \pm 1.57$ & $0.72 \pm 0.28$ & 0.03 \\
\hline 3-sulfo-L-alanine & Amino Acid & $\begin{array}{c}\text { Methionine, Cysteine, SAM and } \\
\text { Taurine Metabolism }\end{array}$ & $1.23 \pm 0.48$ & $0.78 \pm 0.31$ & 0.03 \\
\hline Retinol (Vitamin A) & $\begin{array}{l}\text { Cofactors and } \\
\text { Vitamins }\end{array}$ & Vitamin A Metabolism & $1.37 \pm 0.55$ & $0.88 \pm 0.29$ & 0.037 \\
\hline Arachidate (20:0) & Lipid & $\begin{array}{c}\text { Long Chain Saturated } \\
\text { Fatty Acid }\end{array}$ & $1.25 \pm 0.26$ & $0.97 \pm 0.28$ & 0.041 \\
\hline Palmitoloelycholine & Lipid & $\begin{array}{l}\text { Fatty Acid Metabolism } \\
\text { (Acyl Choline) }\end{array}$ & $2.33 \pm 2.23$ & $0.54 \pm 0.49$ & 0.044 \\
\hline Dihomo-linolenoyl-choline & Lipid & $\begin{array}{l}\text { Fatty Acid Metabolism } \\
\text { (Acyl Choline) }\end{array}$ & $4.06 \pm 4.39$ & $0.74 \pm 0.77$ & 0.04 \\
\hline Dtearoyl ethanolamide & Lipid & Endocannabinoid & $0.88 \pm 0.35$ & $1.21 \pm 0.3$ & 0.045 \\
\hline N-oleoyltaurine & Lipid & Endocannabinoid & $0.57 \pm 0.35$ & $1.11 \pm 0.64$ & 0.042 \\
\hline Glycerophosphorylcholine (GPC) & Lipid & Phospholipid Metabolism & $1.43 \pm 0.58$ & $0.94 \pm 0.32$ & 0.041 \\
\hline $\begin{array}{l}\text { 1-myristoyl-2-palmitoyl-GPC } \\
(14: 0 / 16: 0)\end{array}$ & Lipid & Phosphatidylcholine (PC) & $1.92 \pm 0.79$ & $1.12 \pm 0.6$ & 0.028 \\
\hline $\begin{array}{l}\text { 1-palmitoyl-2-palmitoleoyl-GPC } \\
(16: 0 / 16: 1)\end{array}$ & Lipid & Phosphatidylcholine (PC) & $2.14 \pm 1.17$ & $1.13 \pm 0.48$ & 0.035 \\
\hline 1-palmitoyl-2-linoleoyl-GPC (16:0/18:2) & Lipid & Phosphatidylcholine (PC) & $1.17 \pm 0.11$ & $1 \pm 0.14$ & 0.014 \\
\hline $\begin{array}{l}\text { 1-palmitoyl-2-dihomo-linolenoyl-GPC } \\
\text { (16:0/20:3n3 or } 6)\end{array}$ & Lipid & Phosphatidylcholine (PC) & $1.35 \pm 0.24$ & $1 \pm 0.2$ & 0.003 \\
\hline 1-palmitoyl-2-linoleoyl-GPE (16:0/18:2) & Lipid & Phosphatidylethanolamine (PE) & $1.96 \pm 0.75$ & $1.05 \pm 0.55$ & 0.009 \\
\hline 1-stearoyl-2-linoleoyl-GPE (18:0/18:2) & Lipid & Phosphatidylethanolamine (PE) & $1.9 \pm 0.77$ & $1.14 \pm 0.5$ & 0.025 \\
\hline 1-oleoyl-2-linoleoyl-GPE (18:1/18:2) & Lipid & Phosphatidylethanolamine (PE) & $2.54 \pm 1.18$ & $1.29 \pm 0.73$ & 0.016 \\
\hline 1-palmitoyl-2-oleoyl-GPI (16:0/18:1) & Lipid & Phosphatidylinositol (PI) & $1.97 \pm 0.56$ & $1.33 \pm 0.55$ & 0.026 \\
\hline 1-palmitoyl-2-linoleoyl-GPI (16:0/18:2) & Lipid & Phosphatidylinositol (PI) & $1.83 \pm 0.58$ & $1.23 \pm 0.44$ & 0.026 \\
\hline $\begin{array}{l}\text { 1-palmitoyl-2-arachidonoyl-GPI } \\
(16: 0 / 20: 4)\end{array}$ & Lipid & Phosphatidylinositol (PI) & $1.65 \pm 0.57$ & $1.04 \pm 0.36$ & 0.016 \\
\hline 1-linoleoyl-GPA (18:2) & Lipid & Lysophospholipid & $2.2 \pm 1.04$ & $1.13 \pm 0.62$ & 0.017 \\
\hline 1-palmitoyl-GPC (16:0) & Lipid & Lysophospholipid & $1.17 \pm 0.17$ & $0.93 \pm 0.11$ & 0.003 \\
\hline 2-palmitoyl-GPC (16:0) & Lipid & Lysophospholipid & $1.28 \pm 0.37$ & $0.9 \pm 0.36$ & 0.041 \\
\hline 1-palmitoleoyl-GPC (16:1) & Lipid & Lysophospholipid & $1.79 \pm 0.68$ & $1.01 \pm 0.32$ & 0.006 \\
\hline 2-palmitoleoyl-GPC (16:1) & Lipid & Lysophospholipid & $1.63 \pm 0.9$ & $0.75 \pm 0.47$ & 0.02 \\
\hline 1-palmitoyl-GPI (16:0) & Lipid & Lysophospholipid & $1.92 \pm 0.96$ & $0.86 \pm 0.64$ & 0.015 \\
\hline 1-stearoyl-GPI (18:0) & Lipid & Lysophospholipid & $1.36 \pm 0.43$ & $0.89 \pm 0.5$ & 0.048 \\
\hline 1-linoleoyl-GPI (18:2) & Lipid & Lysophospholipid & $1.54 \pm 0.59$ & $1 \pm 0.49$ & 0.048 \\
\hline $\begin{array}{l}\text { 1-(1-enyl-palmitoyl)-2-palmitoyl-GPC } \\
\text { (P-16:0/16:0) }\end{array}$ & Lipid & Plasmalogen & $0.92 \pm 0.21$ & $1.27 \pm 0.29$ & 0.01 \\
\hline $\begin{array}{l}\text { 1-(1-enyl-palmitoyl)-2-arachidonoyl- } \\
\text { GPC } \\
\text { (P-16:0/20:4) }\end{array}$ & Lipid & Plasmalogen & $0.86 \pm 0.23$ & $1.25 \pm 0.27$ & 0.005 \\
\hline 1-palmitoylglycerol (16:0) & Lipid & Monoacylglycerol & $2.12 \pm 1.1$ & $0.7 \pm 0.31$ & 0.004 \\
\hline 1-palmitoleoylglycerol $(16: 1)$ & Lipid & Monoacylglycerol & $2.6 \pm 2.06$ & $0.72 \pm 0.44$ & 0.026 \\
\hline 1-oleoylglycerol (18:1) & Lipid & Monoacylglycerol & $1.69 \pm 1.01$ & $0.83 \pm 0.5$ & 0.035 \\
\hline 1-linoleoylglycerol (18:2) & Lipid & Monoacylglycerol & $1.65 \pm 0.92$ & $0.85 \pm 0.53$ & 0.037 \\
\hline 1-linolenoylglycerol (18:3) & Lipid & Monoacylglycerol & $1.76 \pm 1$ & $0.86 \pm 0.52$ & 0.034 \\
\hline 1-dihomo-linolenylglycerol (20:3) & Lipid & Monoacylglycerol & $2.69 \pm 2.15$ & $0.87 \pm 0.63$ & 0.027 \\
\hline 2-palmitoylglycerol (16:0) & Lipid & Monoacylglycerol & $1.35 \pm 1.11$ & $0.39 \pm 0.35$ & 0.034 \\
\hline 2-palmitoleoylglycerol $(16: 1)$ & Lipid & Monoacylglycerol & $2.17 \pm 1.99$ & $0.31 \pm 0.51$ & 0.024 \\
\hline 1-heptadecenoylglycerol (17:1) & Lipid & Monoacylglycerol & $1.49 \pm 1.04$ & $0.61 \pm 0.31$ & 0.037 \\
\hline Palmitoyl-oleoyl-glycerol (16:0/18:1) & Lipid & Diacylglycerol & $3.07 \pm 2.59$ & $0.95 \pm 0.8$ & 0.032 \\
\hline Palmitoyl-linoleoyl-glycerol (16:0/18:2) & Lipid & Diacylglycerol & $1.94 \pm 1.26$ & $0.92 \pm 0.53$ & 0.046 \\
\hline $\begin{array}{l}\text { Palmitoyl-docosahexaenoyl-glycerol } \\
(16: 0 / 22: 6)\end{array}$ & Lipid & Diacylglycerol & $1.63 \pm 1.06$ & $0.67 \pm 0.46$ & 0.03 \\
\hline Oleoyl-oleoyl-glycerol (18:1/18:1) & Lipid & Diacylglycerol & $3.03 \pm 2.4$ & $1.09 \pm 0.64$ & 0.044 \\
\hline Sphingomyelin (d17:1/14:0, d16:1/15:0) & Lipid & Sphingomyelins & $1.63 \pm 0.52$ & $1.13 \pm 0.47$ & 0.047 \\
\hline Sphingomyelin (d18:2/24:1, d18:1/24:2) & Lipid & Sphingomyelins & $1.04 \pm 0.2$ & $1.27 \pm 0.23$ & 0.038 \\
\hline 5,6-dihydrouracil & Nucleotide & $\begin{array}{c}\text { Pyrimidine Metabolism, Uracil } \\
\text { containing }\end{array}$ & $0.89 \pm 0.29$ & $1.43 \pm 0.6$ & 0.027 \\
\hline Gamma-glutamylalanine & Peptide & Gamma-glutamyl Amino Acid & $1.57 \pm 0.59$ & $0.82 \pm 0.32$ & 0.004 \\
\hline Gamma-glutamylhistidine & Peptide & Gamma-glutamyl Amino Acid & $1.23 \pm 0.37$ & $0.81 \pm 0.32$ & 0.021 \\
\hline
\end{tabular}


Table 1. Cont.

\begin{tabular}{|c|c|c|c|c|c|}
\hline Biochemical & Super Pathway & Sub Pathway & Control & Ischemic & $p$-Value \\
\hline Gamma-glutamyl-epsilon-lysine & Peptide & Gamma-glutamyl Amino Acid & $1.17 \pm 0.29$ & $0.9 \pm 0.25$ & 0.049 \\
\hline Metabolonic lactone sulfate & $\begin{array}{c}\text { Partially } \\
\text { Characterized } \\
\text { Molecules }\end{array}$ & $\begin{array}{c}\text { Partially } \\
\text { Characterized Molecules }\end{array}$ & $2 \pm 1.34$ & $0.47 \pm 0.49$ & 0.009 \\
\hline 4-allylcatechol sulfate & Xenobiotics & Benzoate Metabolism & $1.21 \pm 0.79$ & $0.52 \pm 0.42$ & 0.032 \\
\hline S-allylcysteine & Xenobiotics & Food Component/Plant & $2.49 \pm 2.47$ & $0.32 \pm 0.38$ & 0.03 \\
\hline 2,6-dihydroxybenzoic acid & Xenobiotics & Drug-Topical Agents & $2.31 \pm 1.2$ & $1 \pm 0.58$ & 0.009 \\
\hline Thioproline & Xenobiotics & Chemical & $1.13 \pm 0.36$ & $0.8 \pm 0.27$ & 0.048 \\
\hline
\end{tabular}

Note: A pairwise comparison was performed between the male and female stroke patients to identify distinct biomarkers associated with male and female patients among the thousands of variables using Student $T$-tests. The level of significance was set at $p<0.05$.

Table 2. A summary of the detailed results from the pathway analysis for female patients. We tested many pathways at the same time, and the statistical $p$ values from enrichment analysis were further adjusted for multiple testings. From the table, the "Total" in the table is the total number of compounds in the pathway; the "Hits" is the actually matched number from the user uploaded data; the "Raw $\mathrm{p}$ " is the original $\mathrm{p}$ value calculated from the enrichment analysis; the "Holm $\mathrm{p}$ " is the $\mathrm{p}$ value adjusted by Holm-Bonferroni method; the "FDR p" is the p value adjusted using false discovery rate; the "Impact" is the pathway impact value calculated from pathway topology analysis.

\begin{tabular}{lcccccccc}
\hline \multicolumn{1}{c}{ Metabolites } & Total & Expected & Hits & Raw p & $-\log 10(\mathbf{p})$ & Holm Adjust & FDR & Impact \\
\hline Glycerophospholipid metabolism & 36 & 0.37 & 3 & $5.24 \times 10^{-3}$ & $2.28 \times 10^{0}$ & $4.40 \times 10^{-1}$ & $4.40 \times 10^{1}$ & 0.25 \\
Pantothenate and CoA biosynthesis & 19 & 0.20 & 2 & $1.54 \times 10^{-2}$ & $1.81 \times 10^{0}$ & $1.00 \times 10^{0}$ & $5.24 \times 10^{1}$ & 0.05 \\
beta-Alanine metabolism & 21 & 0.22 & 2 & $1.87 \times 10^{-2}$ & $1.73 \times 10^{0}$ & $1.00 \times 10^{0}$ & $5.24 \times 10^{1}$ & 0.16 \\
Linoleic acid metabolism & 5 & 0.05 & 1 & $5.06 \times 10^{-2}$ & $1.30 \times 10^{0}$ & $1.00 \times 10^{0}$ & $9.96 \times 10^{1}$ & 0.00 \\
Pyrimidine metabolism & 39 & 0.40 & 2 & $5.93 \times 10^{-2}$ & $1.23 \times 10^{0}$ & $1.00 \times 10^{0}$ & $9.96 \times 10^{1}$ & 0.04 \\
alpha-Linolenic acid metabolism & 13 & 0.13 & 1 & $1.27 \times 10^{-1}$ & $8.97 \times 10^{-1}$ & $1.00 \times 10^{0}$ & $1.00 \times 10^{0}$ & 0.00 \\
Glycerolipid metabolism & 16 & 0.17 & 1 & $1.54 \times 10^{-1}$ & $8.13 \times 10^{-1}$ & $1.00 \times 10^{0}$ & $1.00 \times 10^{0}$ & 0.01 \\
Selenocompound metabolism & 20 & 0.21 & 1 & $1.88 \times 10^{-1}$ & $7.25 \times 10^{-1}$ & $1.00 \times 10^{0}$ & $1.00 \times 10^{0}$ & 0.00 \\
Alanine, aspartate and & 28 & 0.29 & 1 & $2.54 \times 10^{-1}$ & $5.95 \times 10^{-1}$ & $1.00 \times 10^{0}$ & $11.00 \times 10^{0}$ & 0.00 \\
glutamate metabolism & & & & & & & \\
Phosphatidylinositol & 28 & 0.29 & 1 & $2.54 \times 10^{-1}$ & $5.95 \times 10^{-1}$ & $1.00 \times 10^{0}$ & $1.00 \times 10^{0}$ & 0.00 \\
signaling system & 36 & 0.37 & 1 & $3.15 \times 10^{-1}$ & $5.02 \times 10^{-1}$ & $1.00 \times 10^{0}$ & $1.00 \times 10^{0}$ & 0.00 \\
Arachidonic acid metabolism & 36 & 0.37 & 1 & $3.15 \times 10^{-1}$ & $5.02 \times 10^{-1}$ & $1.00 \times 10^{0}$ & $1.00 \times 10^{0}$ & 0.00 \\
Biosynthesis of unsaturated & 41 & 0.42 & 1 & $3.50 \times 10^{-1}$ & $4.56 \times 10^{-1}$ & $1.00 \times 10^{0}$ & $1.00 \times 10^{0}$ & 0.01 \\
fatty acids & 48 & 0.50 & 1 & $3.97 \times 10^{-1}$ & $4.01 \times 10^{-1}$ & $1.00 \times 10^{0}$ & $1.00 \times 10^{0}$ & 0.00 \\
Tryptophan metabolism & & & & & & &
\end{tabular}

Notably, 1-(1-enyl-palmitoyl)-2-arachidonoyl-GPC (P-16:0/20:4) $(\mathrm{AUC}=0.914$, 0.765-1.000), 1-(1-enyl-palmitoyl)-2-palmitoyl-GPC (P-16:0/16:0) $(\mathrm{AUC}=0.840,0.656-$ 1.000), and 5,6-dihydrouracil (P-16:0/20:2) (AUC $=0.815,0.601-1.000)$ were all found to be significant predictors in female ischemic stroke patients. The AUC of the optimized model was 0.945 (95\% CI: 0.875-0.956) in the training set and 0.845 (95\% CI: 0.721-0.923) in the validation set. None of the other metabolites were included in the model because of multicolinearity in the information provided by these compounds, and three metabolites contributed to the combined model. The constructed receiver operating characteristic (ROC) curve for the three individual metabolites is presented in Figure 3.

The PCA did not reveal separation between male patients' ischemic and control groups based on factor-reducing components (Figure 4). The control selection, that was not originally designed for metabolomics study may introduce selection bias, especially if not properly accounted for. This could induce the bias in the metabolite-phenotype relationships in selected groups and affect the results. Component 1 encompassed $23.890 \%$ of the variance, and Component 2 encompassed $12.218 \%$ of the data. 39 metabolite levels were significantly different between the ischemic and control group in the male cohort (Table 3). Biological pathway analysis for male patients revealed five metabolic pathways (Figure 5 and Table 4), including valine, leucine, and isoleucine biosynthesis, valine, leucine, and isoleucine degradation, pantothenate and CoA biosynthesis, primary bile acid biosynthesis, and steroid hormone biosynthesis. Significant metabolites that were predictive of 
male ischemic patients were 5alpha-androstan-3alpha,17beta-diol disulfate (AUC $=0.951$, $0.857-1.000$ ), alpha-hydroxyisocaproate (AUC $=0.938,0.832-1.000$ ), threonate (AUC $=0.877$, $0.716-1.000$ ), and bilirubin (AUC $=0.817,0.746-1.000$ ). The AUC of the optimized model was 0.913 (95\% CI: 0.825-0.915 in the training set and 0.845 (95\% CI: 0.7016-0.9015) in the validation set. The ROC curve for the four metabolites is presented in Figure 6.

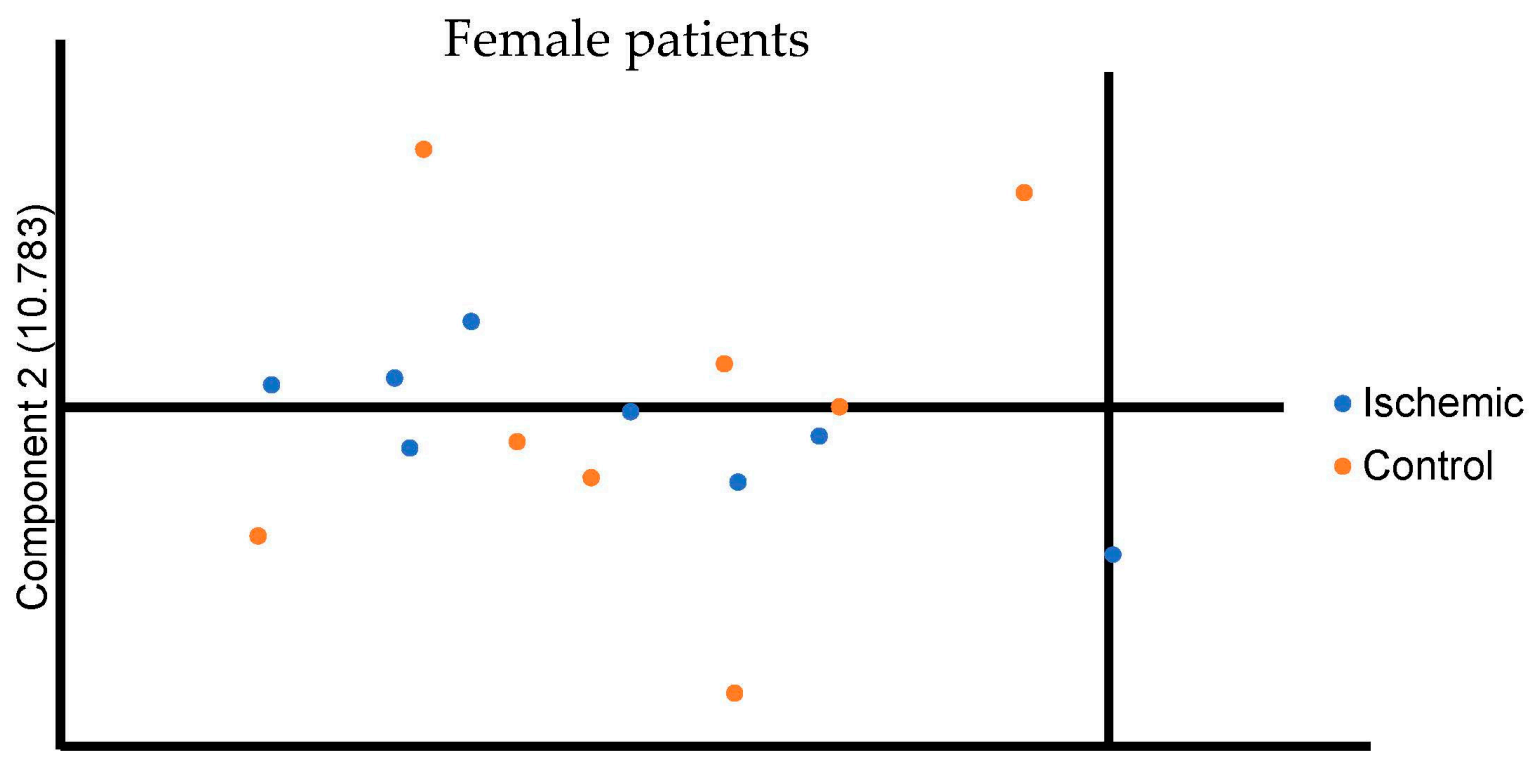

Component $1(21.399 \%)$

Figure 1. Principal Component Analysis for all metabolites in the female ischemic and control population. Component 1 is plotted on the $x$ axis and Component 2 is plotted on the $\mathrm{Y}$ axis. Component 1 encompassed 21.399\% of the variation between participants and Component 2 encompassed $10.783 \%$ of the variation between participants.

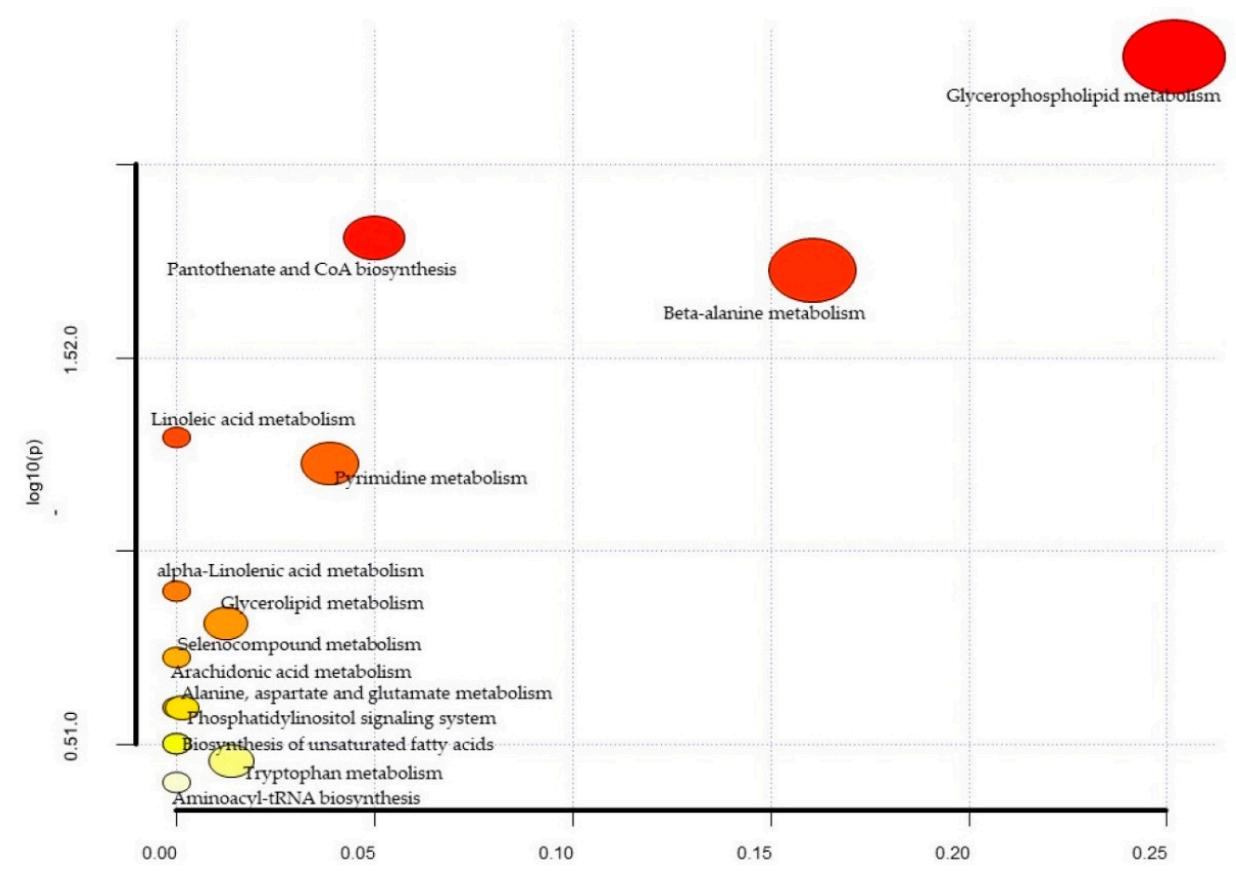

Figure 2. Metabolic pathway analysis for female patients. The $x$-axis represents the pathway impact, while the $y$-axis represents the $-\log (p)$. The analyzed pathway showed the metabolic network reprogramming of female ischemic patients with detailed impact. 
Female

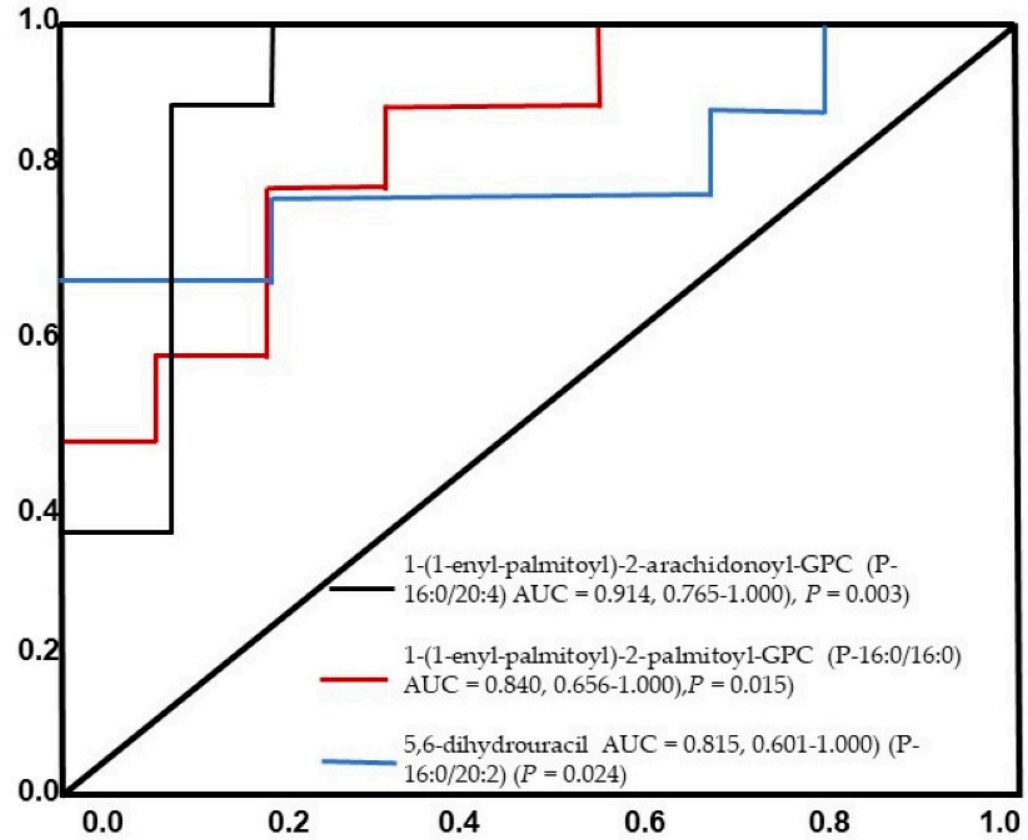

Figure 3. The ROC analysis results from the four diagnostic models calculated from the logistic regression analysis for female patients. The performance of each biomarker model was evaluated by the area under the ROC curve (AUC) and the determination of sensitivity and specificity at the optimal cut-off point defined by the minimum distance to the top-left corner.

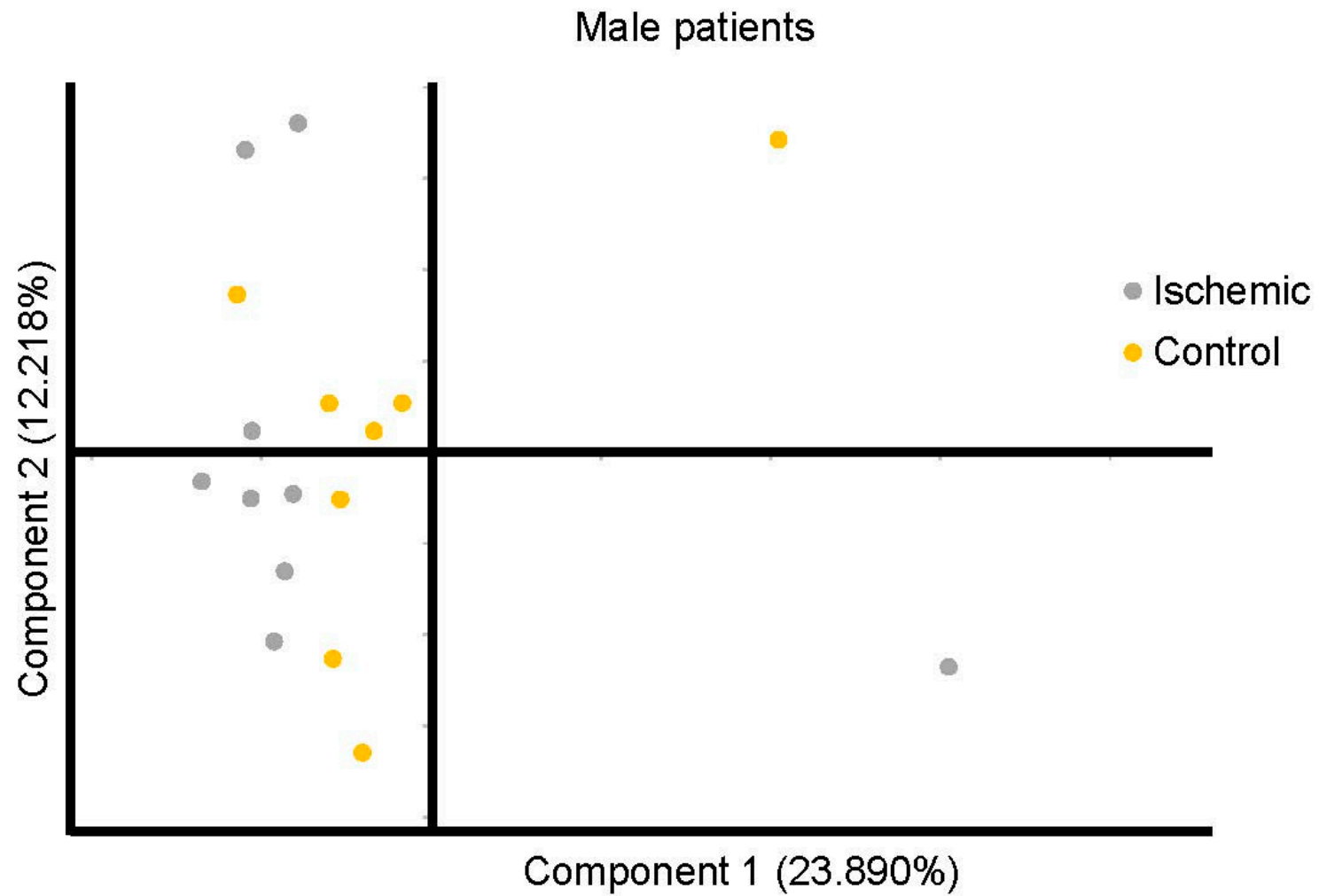

Figure 4. Principal Component Analysis for all metabolites in the male ischemic and control population. Component 1 is plotted on the $\times$ axis and Component 2 is plotted on the $Y$ axis. Component 1 encompassed 23.890\% of the variation between participants and Component 2 encompassed $12.218 \%$ of the variation between participants. 
Table 3. Metabolite differences between ischemic stroke patients and control patients in the male population.

\begin{tabular}{|c|c|c|c|c|c|}
\hline Biochemical. & Super Pathway & Sub Pathway & Control & Ischemic & $p$-Value \\
\hline N6-acetyllysine & Amino Acid & Lysine Metabolism & $1.13 \pm 0.34$ & $0.69 \pm 0.21$ & 0.004 \\
\hline Fructosyllysine & Amino Acid & Lysine Metabolism & $0.79 \pm 0.25$ & $1.52 \pm 0.93$ & 0.037 \\
\hline 4-methyl-2-oxopentanoate & Amino Acid & $\begin{array}{l}\text { Leucine, Isoleucine and } \\
\text { Valine Metabolism }\end{array}$ & $0.8 \pm 0.32$ & $1.73 \pm 1.07$ & 0.033 \\
\hline Alpha-hydroxyisocaproate & Amino Acid & $\begin{array}{l}\text { Leucine, Isoleucine and } \\
\text { Valine Metabolism }\end{array}$ & $0.71 \pm 0.3$ & $1.75 \pm 0.68$ & 0.001 \\
\hline 3-methyl-2-oxovalerate & Amino Acid & $\begin{array}{l}\text { Leucine, Isoleucine and } \\
\text { Valine Metabolism }\end{array}$ & $0.77 \pm 0.37$ & $1.41 \pm 0.77$ & 0.045 \\
\hline 3-methyl-2-oxobutyrate & Amino Acid & $\begin{array}{l}\text { Leucine, Isoleucine and } \\
\text { Valine Metabolism }\end{array}$ & $0.89 \pm 0.25$ & $1.36 \pm 0.57$ & 0.048 \\
\hline N-acetylmethionine sulfoxide & Amino Acid & $\begin{array}{c}\text { Methionine, Cysteine, SAM and } \\
\text { Taurine Metabolism }\end{array}$ & $1.81 \pm 1.27$ & $0.59 \pm 0.24$ & 0.02 \\
\hline Threonate & $\begin{array}{l}\text { Cofactors } \\
\text { and Vitamins }\end{array}$ & $\begin{array}{c}\text { Ascorbate and } \\
\text { Aldarate Metabolism }\end{array}$ & $0.58 \pm 0.34$ & $1.12 \pm 0.3$ & 0.003 \\
\hline Oxalate (ethanedioate) & $\begin{array}{l}\text { Cofactors } \\
\text { and Vitamins }\end{array}$ & $\begin{array}{c}\text { Ascorbate and } \\
\text { Aldarate Metabolism }\end{array}$ & $0.53 \pm 0.36$ & $1.17 \pm 0.42$ & 0.003 \\
\hline Bilirubin $(\mathrm{E}, \mathrm{Z}$ or $\mathrm{Z}, \mathrm{E})$ & $\begin{array}{l}\text { Cofactors } \\
\text { and Vitamins }\end{array}$ & $\begin{array}{c}\text { Hemoglobin and } \\
\text { Porphyrin Metabolism }\end{array}$ & $1.03 \pm 0.65$ & $1.79 \pm 0.85$ & 0.049 \\
\hline Erucate $(22: 1 n 9)$ & Lipid & $\begin{array}{l}\text { Long Chain Monounsaturated } \\
\text { Fatty Acid }\end{array}$ & $0.92 \pm 0.28$ & $1.2 \pm 0.28$ & 0.049 \\
\hline Linolenoylcarnitine (C18:3) & Lipid & $\begin{array}{l}\text { Fatty Acid Metabolism (Acyl } \\
\text { Carnitine, Polyunsaturated) }\end{array}$ & $0.77 \pm 0.42$ & $1.23 \pm 0.5$ & 0.049 \\
\hline 3-hydroxyoleoylcarnitine & Lipid & $\begin{array}{c}\text { Fatty Acid Metabolism (Acyl } \\
\text { Carnitine, Hydroxy) }\end{array}$ & $0.7 \pm 0.4$ & $1.18 \pm 0.54$ & 0.047 \\
\hline 3-hydroxydecanoate & Lipid & Fatty Acid, Monohydroxy & $0.86 \pm 0.38$ & $1.39 \pm 0.5$ & 0.021 \\
\hline 3-hydroxylaurate & Lipid & Fatty Acid, Monohydroxy & $0.76 \pm 0.45$ & $1.38 \pm 0.61$ & 0.026 \\
\hline 3-hydroxymyristate & Lipid & Fatty Acid, Monohydroxy & $0.74 \pm 0.31$ & $1.41 \pm 0.56$ & 0.006 \\
\hline 3-hydroxyoleate & Lipid & Fatty Acid, Monohydroxy & $0.75 \pm 0.41$ & $1.95 \pm 1.2$ & 0.018 \\
\hline 1-linoleoyl-GPG (18:2) & Lipid & Lysophospholipid & $0.71 \pm 0.35$ & $1.18 \pm 0.36$ & 0.013 \\
\hline $\begin{array}{l}\text { Glycosyl ceramide }(\mathrm{d} 18: 2 / 24: 1 \text {, } \\
\mathrm{d} 18: 1 / 24: 2)\end{array}$ & Lipid & Hexosylceramides (HCER) & $1.2 \pm 0.42$ & $0.76 \pm 0.23$ & 0.014 \\
\hline $\begin{array}{l}\text { 5alpha-pregnan-3beta,20alpha-diol } \\
\text { monosulfate ( } 2 \text { ) }\end{array}$ & Lipid & Progestin Steroids & $0.51 \pm 0.4$ & $1.14 \pm 0.71$ & 0.039 \\
\hline $\begin{array}{l}\text { 5alpha-pregnan-3beta,20alpha- } \\
\text { diol disulfate }\end{array}$ & Lipid & Progestin Steroids & $0.78 \pm 0.55$ & $1.54 \pm 0.58$ & 0.013 \\
\hline Cortisone & Lipid & Corticosteroids & $0.67 \pm 0.43$ & $1.13 \pm 0.34$ & 0.024 \\
\hline $\begin{array}{l}\text { Androstenediol (3beta,17beta) } \\
\text { monosulfate (1) }\end{array}$ & Lipid & Androgenic Steroids & $0.67 \pm 0.41$ & $2.46 \pm 1.87$ & 0.021 \\
\hline $\begin{array}{l}\text { Androstenediol } \\
\text { (3beta,17beta) disulfate }\end{array}$ & Lipid & Androgenic Steroids & $1.13 \pm 0.99$ & $2.39 \pm 0.98$ & 0.015 \\
\hline $\begin{array}{l}\text { Androstenediol (3alpha,17alpha) } \\
\text { monosulfate }\end{array}$ & Lipid & Androgenic Steroids & $0.87 \pm 0.75$ & $2.48 \pm 2.02$ & 0.039 \\
\hline $\begin{array}{l}\text { 5alpha-androstan-3alpha,17beta- } \\
\text { diol disulfate }\end{array}$ & Lipid & Androgenic Steroids & $0.63 \pm 0.39$ & $3.06 \pm 2.51$ & 0.02 \\
\hline $\begin{array}{l}\text { 5alpha-androstan-3alpha,17beta-diol } \\
\text { 17-glucuronide }\end{array}$ & Lipid & Androgenic Steroids & $0.72 \pm 0.74$ & $1.58 \pm 0.89$ & 0.041 \\
\hline $\begin{array}{l}\text { 5alpha-androstan-3beta,17beta- } \\
\text { diol disulfate }\end{array}$ & Lipid & Androgenic Steroids & $1.26 \pm 1.65$ & $3.95 \pm 2.22$ & 0.01 \\
\hline Glycochenodeoxycholate & Lipid & Primary Bile Acid Metabolism & $1.44 \pm 0.94$ & $0.51 \pm 0.53$ & 0.022 \\
\hline Glyco-beta-muricholate & Lipid & Primary Bile Acid Metabolism & $1.32 \pm 1.32$ & $0.11 \pm 0.06$ & 0.026 \\
\hline Glycodeoxycholate & Lipid & Secondary Bile Acid Metabolism & $1.93 \pm 1.77$ & $0.19 \pm 0.36$ & 0.018 \\
\hline Taurodeoxycholate & Lipid & Secondary Bile Acid Metabolism & $1.81 \pm 1.9$ & $0.18 \pm 0.16$ & 0.033 \\
\hline Glycodeoxycholate 3-sulfate & Lipid & Secondary Bile Acid Metabolism & $1.39 \pm 0.98$ & $0.55 \pm 0.58$ & 0.044 \\
\hline Gamma-glutamylphenylalanine & Peptide & Gamma-glutamyl Amino Acid & $1.13 \pm 0.32$ & $0.82 \pm 0.29$ & 0.048 \\
\hline Gamma-glutamyltryptophan & Peptide & Gamma-glutamyl Amino Acid & $1.07 \pm 0.41$ & $0.72 \pm 0.22$ & 0.04 \\
\hline Saccharin & Xenobiotics & Food Component/Plant & $1.17 \pm 1.37$ & $0.06 \pm 0$ & 0.042 \\
\hline 4-acetamidophenylglucuronide & Xenobiotics & Drug-Analgesics, Anesthetics & $0.84 \pm 0.7$ & $0.26 \pm 0.29$ & 0.043 \\
\hline $\begin{array}{l}\text { 2-methoxyacetaminophen } \\
\text { glucuronide }\end{array}$ & Xenobiotics & Drug_Analgesics, Anesthetics & $1.09 \pm 1.06$ & $0.13 \pm 0.1$ & 0.027 \\
\hline 3-(methylthio)acetaminophen sulfate & Xenobiotics & Drug-Analgesics, Anesthetics & $1.97 \pm 2.25$ & $0.04 \pm 0.07$ & 0.033 \\
\hline
\end{tabular}

Note: A pairwise comparison was performed between the male and female stroke patients to identify distinct biomarkers associated with male and female patients among the thousands of variables using Student $T$-tests. The level of significance was set at $p<0.05$. 


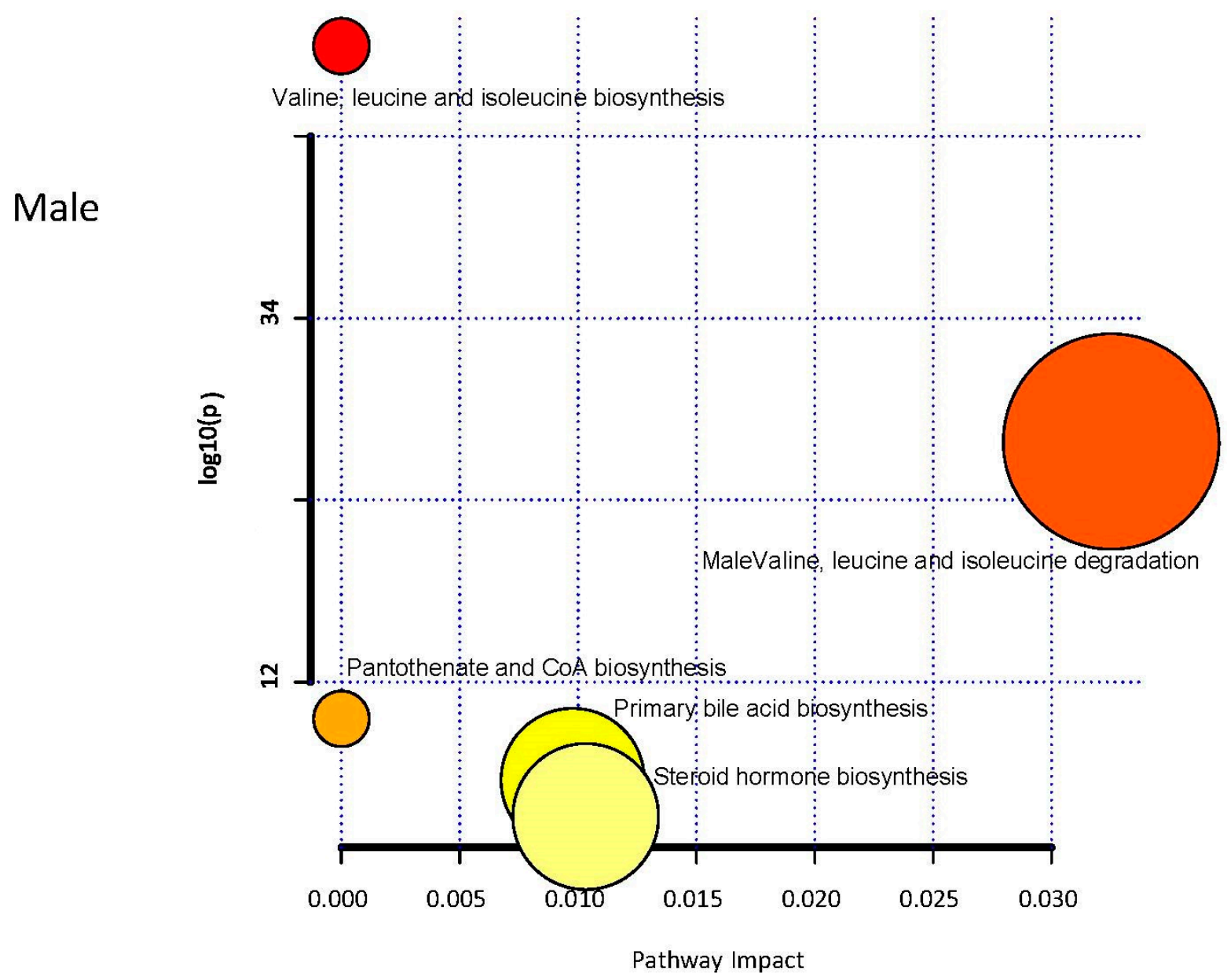

Figure 5. Metabolic pathway analysis for male patients. The $x$-axis represents the pathway impact, while the $y$-axis represents the $-\log (p)$. The analyzed pathway showed the metabolic network reprogramming of male ischemic patients with detailed impact.

Male

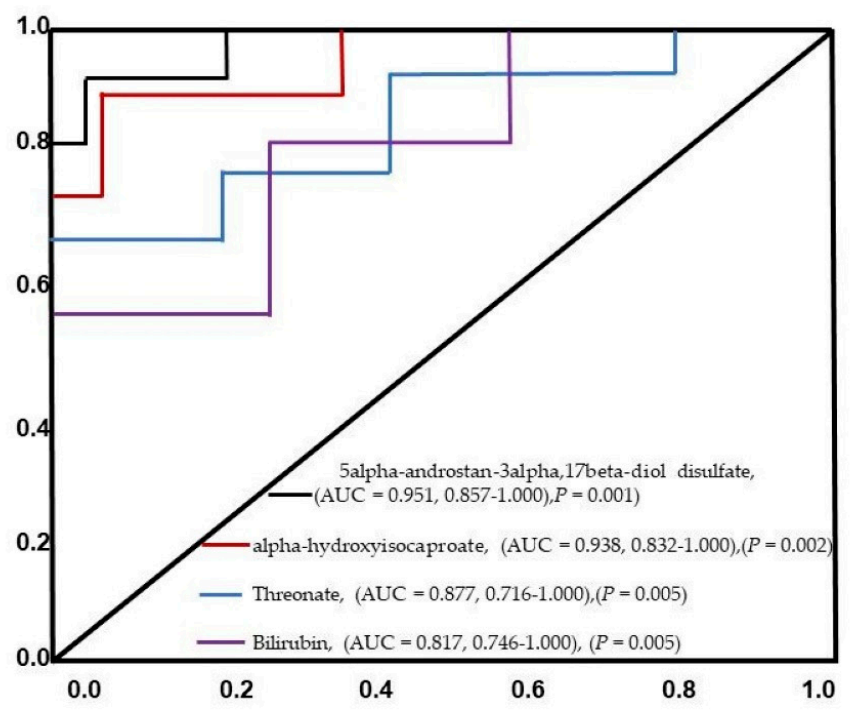

Figure 6. The ROC analysis results from the four diagnostic models calculated from the logistic regression analysis for male patients. The performance of each biomarker model was evaluated by the area under the ROC curve (AUC) and the determination of sensitivity and specificity at the optimal cut-off point defined by the minimum distance to the top-left corner. 
Table 4. Detailed results from the pathway analysis for male patients. From the table, the "Total" in the table is the total number of compounds in the pathway; the "Hits" is the actually matched number from the user uploaded data; the "Raw $\mathrm{p}$ " is the original $\mathrm{p}$ value calculated from the enrichment analysis; the "Holm $\mathrm{p}$ " is the $\mathrm{p}$ value adjusted by Holm-Bonferroni method; the "FDR p" is the $\mathrm{p}$ value adjusted using false discovery rate; the "Impact" is the pathway impact value calculated from pathway topology analysis.

\begin{tabular}{ccccccccc}
\hline & Total & Expected & Hits & Raw p & $-\log 10(\mathbf{p})$ & Holm Adjust & FDR & Impact \\
\hline $\begin{array}{c}\text { Valine, leucine and } \\
\text { isoleucine biosynthesis }\end{array}$ & 8 & 0.07 & 3 & $3.20 \times 10^{-5}$ & $4.49 \times 10^{0}$ & $2.69 \times 10^{-3}$ & $2.69 \times 10^{-3}$ & 0.00 \\
$\begin{array}{c}\text { Valine, leucine and } \\
\text { isoleucine degradation }\end{array}$ & 40 & 0.36 & 3 & $4.76 \times 10^{3}$ & $2.32 \times 10^{0}$ & $3.95 \times 10^{-1}$ & $2.00 \times 10^{-1}$ & 0.03 \\
$\quad \begin{array}{c}\text { Pantothenate and } \\
\text { CoA biosynthesis }\end{array}$ & 19 & 0.17 & 1 & $1.59 \times 10^{-1}$ & $7.98 \times 10^{-1}$ & $1.00 \times 10^{0}$ & $1.00 \times 10^{0}$ & 0.00 \\
$\begin{array}{c}\text { Primary bile acid biosynthesis } \\
\text { Steroid hormone biosynthesis }\end{array}$ & 46 & 0.42 & 1 & $3.45 \times 10^{-1}$ & $4.62 \times 10^{-1}$ & $1.00 \times 10^{0}$ & $1.00 \times 10^{0}$ & 0.01 \\
\hline
\end{tabular}

\section{Discussion}

Computer tomography (CT) scans can resolve some aspects of stroke onset, and behavioral evaluations of diagnosis are entirely symptom-based, decreasing diagnostic reliability and hindering treatment [44]. Blood biomarkers related to stroke would provide an objective measurement to inform clinical assessments and treatment decisions [45]. There is no single biomarker that directly captures all the pathophysiology of stroke [45]. Therefore, using a metabolomics approach to analyze metabolites holds promise to capture the complex pathophysiological processes of AIS. This is because the metabolomics approach monitors alterations in metabolites in ways that formal identification of a single biomarker does not capture [46]. This in turn allows for the identification of metabolic changes that drive pathology.

While gender differences in risk factors of stroke has been investigated [47,48], differences in metabolites among male and female patients with AIS is not fully understood. This study uses biological samples from AIS patients from a large, carefully phenotyped epidemiologic patient cohort to identify distinctive metabolic signatures in men and women AIS patients. First, we detected 49 distinct metabolites and 15 metabolic pathways for women and 39 metabolites and 5 metabolic pathways that exhibited reprogramming. Our logistic regression and ROC analysis reveal 1-(1-enyl-palmitoyl)-2-arachidonoyl-GPC, 1(1-enyl-palmitoyl)-2-palmitoyl-GPC, and 5,6-dihydrouracil to be significant predictors for female AIS patients. Moreover, 5alpha-androstan-3alpha,17beta-diol disulfate, alphahydroxyisocaproate, threonate, and bilirubin were associated with male AIS patients.

Glycerophosphocholine (GPC) metabolites modulate atherosclerosis and are associated with many risk factors [49]. GPC metabolites are platelet activating metabolites that modulate systemic oxidative stress and inflammation [50]. Findings from recent studies [51-53] indicate that GPC metabolites may improve the prediction of outcomes in different clinical conditions. This finding supports our current results that GPCs, including 1-(1-enyl-palmitoyl)-2-arachidonoyl-GPC and 1-(1-enyl-palmitoyl)-2-palmitoyl-GPC identified in this study, maybe sensitive indicators that can be used to help improve our understanding of the pathobiology of stroke in female patients.

Dihydrouracil (5,6-Dihydrouracil) is a metabolite of uracil that has been used as a marker to identify dihydropyrimidine dehydrogenase (DPD)-deficient [54]. Deficiencies in DPD activity are associated with reducing 5,6-Dihydrouracil catabolism, which can lead to severe toxicity in different clinical conditions [55]. Since DPD deficiency impairs the metabolic breakdown of 5,6-Dihydrouracil, the accumulation of this uracil can be detected in the plasma of patients with specific clinical conditions [56]. Therefore, identifying toxicity in stroke patients has the potential to significantly improve patient care using 5,6-Dihydrouracil as a marker. Moreover, since deficiency in enzymes downstream of DPD, such as dihydropyrimidase (DHP) and /or $\beta$-ureidopropionase (UDP), could alter the 5,6-Dihydrouracil catabolic pathway [57], our finding supports the possibility that DPD 
function could indicate an error of 5,6-Dihydrouracil metabolism detected in metabolomic analysis in our female ischemic stroke patients [54]. This possibility is supported by other studies [58,59] that DPD function could indicate an error of 5,6-Dihydrouracil metabolism.

5 alpha-Androstane- 3 alpha 17 beta-diol is a testosterone metabolite. The modulatory role of testosterone was initially thought to be via 5 alpha reduction to the potent androgen dihydrotestosterone (DHT) and its subsequent binding to the androgen receptor [60]. However, DHT is metabolized to the estrogen receptor beta-isoform (ERbeta) agonist, $5 \alpha$-androstane $3 \beta, 17 \beta$ Diol (3 $\beta$-Diol) [61]. This finding suggests that $3 a / b$-diol also represent potential precursors of DHT, and that the back conversion of DHT from $3 \alpha$-and $3 \beta$-diol can represent a promising target in maintaining hormonal homeostasis in male ischemic stroke patients.

Alpha-hydroxyisocaproate (HICA) is derived from leucine metabolism in the human connective tissue [62]. HICA helps protect against muscle breakdown due to its anti-catabolic properties by protecting against excessive muscle damage and protein breakdown $[63,64]$. Elevated levels of HICA have been reported in the urine of patients with dihydrolipoyl dehydrogenase deficiency [65]. Our finding of significant levels of HICA in male AIS, suggests that HICA may be linked with ischemic stroke among male patients. In the connective tissue where HICA is well expressed, the HICA functions are not fully understood [66]. A relatively low basal protein synthesis caused by pretreatment with HICA is reported to suppress inflammatory responses of iNOS, IL-6, and ubiquitin-proteasome system's downregulation [67]. In general, HICA is proposed to improve systemic inflammation because AMPK activation generally suppresses inflammation in several tissues [68]. The suppression of systemic inflammation results in increased energy efficiency and a decrease in proinflammatory cytokines [69]. It is also possible that systemic effects of HICA may be associated with systemic inflammation among male AIS patients. A future study on HICA among male AIS patients will help elucidate how the health benefits of HICA may contribute to the extension of healthy life in AIS patients.

Bilirubin is an end product of heme metabolism, and higher levels of serum bilirubin have been proposed to offer therapeutic advantages in oxidative stress-mediated diseases [70]. Blirubin possesses potent antioxidant properties [71], indicating that higher levels of serum bilirubin might provide a therapeutic advantage in oxidative stress-mediated conditions including stroke [72]. Mechanisms of oxidative stress-related neuronal death are associated with oxidative stress linked to pathogenesis of stroke [72]. This finding indicates that elevated serum bilirubin levels may reflect the intensity of oxidative stress [73]. In this study, we found that higher serum bilirubin was associated with male stroke patients. Therefore, male stroke patients may provide an opportunity to examine further the role of bilirubin in the pathophysiology related to oxidative stress in stroke patients. This may offer therapeutic avenues to limit the damage from a stroke facilitated by oxidative stress that results in neuronal loss.

Magnesium L-Threonate is a novel form of magnesium, and magnesium is a critical cofactor for many enzymes involved in glucose metabolism, protein production, and nucleic acid synthesis [74]. Therefore, magnesium deficiency is associated with many diseases, such as Alzheimer's, asthma, attention deficit hyperactivity disorder, type 2 diabetes, hypertension, and stroke [75]. Moreover, magnesium intake is reported to have an inverse association with total and ischemic stroke in a dose-response pattern [76]. Our finding of an association of magnesium with male ischemic stroke patients suggests the optimization of magnesium for stroke prevention or management of stroke. This can improve not only population-wide cardiovascular health but also initiate dietary healthcare to prevent stroke.

Several studies have investigated different metabolites in stroke [26,28,32,33]. Findings reveal new metabolites pathways for ischemic stroke subtypes and provide a new avenue to explore the pathophysiological mechanisms underlying ischemic stroke and its subtypes. A recent study by Daokun et al. [13] that focused on men and women stroke patients identified circulating biomarkers for stroke and novel pathways for AIS and its subtypes. 
The results reveal promising blood-based biomarkers and novel etiologic pathways of ischemic stroke, but the study did not focus on differences in metabolites between men and women AIS patients. While findings from existing studies [13,26-34] highlight the potential of metabolomics for discovering novel circulating biomarkers for stroke and its subtypes, most of the studies did not report gender-differentiated results. Therefore, the specific metabolites and related pathways that are directly associated with men and women AIS patients are not fully understood. As a basis for a gender-specific care for AIS patients, the characterization of differences in metabolites between the men and women AIS patients in the current study might lead to concrete starting points for a future research to improve care for men and men AIS patients.

\section{Conclusions}

The need to improve the diagnosis of stroke for both male and female patients has motivated the use of omic-based approaches to identify novel markers for stroke biology and biomarker development. Although several markers have shown promise, none has been translated into clinical practice. Ischemic stroke is a heterogeneous clinical condition and a single biomarker may not be able to capture the complicated pathophysiology changes associated with stroke in both male and female patients. In the current study, we identified (1-enyl-palmitoyl)-2-arachidonoyl-GPC, 1-(1-enyl-palmitoyl)-2-palmitoyl-GPC and 5,6-dihydrouracil as metabolites that are predictive of ischemic stroke among women while 5alpha-androstan-3alpha,17beta-diol disulfate, alpha-hydroxyisocaproate, threonate and bilirubin predicted ischemic stroke in men patients. Our findings highlight the potential of metabolomics to reveal new pathways for IS and provide a new avenue to explore IS's pathophysiological mechanisms among men and women ischemic stroke patients.

Author Contributions: N.P., R.C., S.A., R.R. and T.I.N. designed the concept, experimental design and data analysis, while R.L.G. critically revised the drafts, interprets the results, read and approved the last version of this manuscript. All authors have read and agreed to the published version of the manuscript.

Funding: This study was funded by NIH R25 Grant (1 R25 GM123935-01A1).

Institutional Review Board Statement: The study was conducted according to the guidelines of the Declaration of Helsinki, and approved by the Institutional Review Board (or Ethics Committee) of NAME OF INSTITUTE (Pro00072801 and date of approval is 10/6/2020.

Informed Consent Statement: Informed consent was obtained from all subjects involved in the study.

Data Availability Statement: The data presented in this study are available on request from the corresponding author.

Acknowledgments: We thank the stroke unit of PRISMA Health system for helping in the sample.

Conflicts of Interest: The authors declare no conflict of interest.

\section{References}

1. Bushnell, C.D.; Chaturvedi, S.; Gage, K.R.; Herson, P.S.; Hurn, P.D.; Jiménez, M.C.; Kittner, S.J.; Madsen, T.E.; McCullough, L.D.; McDermott, M.; et al. Sex differences in stroke: Challenges and opportunities. J. Cereb. Blood Flow Metab. 2018, 38, $2179-2191$. [CrossRef]

2. Avan, A.; Digaleh, H.; Di Napoli, M.; Stranges, S.; Behrouz, R.; Shojaeianbabaei, G.; Amiri, A.; Tabrizi, R.; Mokhber, N.; Spence, J.D.; et al. Socioeconomic status and stroke incidence, prevalence, mortality, and worldwide burden: An ecological analysis from the Global Burden of Disease Study 2017. BMC Med. 2019, 17, 1-30. [CrossRef]

3. Persky, R.W.; Turtzo, L.C.; McCullough, L.D. Stroke in Women: Disparities and Outcomes. Curr. Cardiol. Rep. 2010, $12,6-13$. [CrossRef] [PubMed]

4. $\quad$ Blum, B.; Penwell, A.; Wormack, L.; Walker, B.; Lari, S.; Nathaniel, T.I. Gender and thrombolysis therapy in acute ischemic stroke patients with incidence of obesity. Neurol. Sci. 2019, 40, 1829-1839. [CrossRef] [PubMed]

5. Gainey, J.; Brechtel, L.; Konklin, S.; Madeline, L.; Lowther, E.; Blum, B.; Nathaniel, T.I. In a stroke cohort with incident hypertension; are more women than men likely to be excluded from recombinant tissue-type Plasminogen Activator (rtPA)? J. Neurol. Sci. 2018, 387, 139-146. [CrossRef] [PubMed]

6. Hiraga, A. Gender Differences and Stroke Outcomes. Neuroepidemiology 2017, 48, 61-62. [CrossRef] [PubMed] 
7. Gainey, J.; Wormack, L.; Brechtel, L.; Nathaniel, T.I. Abstract WP89: A Functional Outcome Model for a Telestroke-guided Tissue Plasminogen Activator Treatment of Stroke Patients. Stroke 2018, 49, 89. [CrossRef]

8. Wassertheil-Smoller, S. Stroke in Women. Nutr. Metab. Cardiovasc. Dis. 2010, 20, 419-425. [CrossRef]

9. Reeves, M.J.; Bushnell, C.D.; Howard, G.; Gargano, J.W.; Duncan, P.; Lynch, G.; Khatiwoda, A.; Lisabeth, L. Sex differences in stroke: Epidemiology, clinical presentation, medical care, and outcomes. Lancet Neurol. 2008, 7, 915-926. [CrossRef]

10. Nathaniel, T.I.; Gainey, J.; Blum, B.; Montgomery, C.; Ervin, L.; Madeline, L. Clinical Risk Factors in Thrombolysis therapy: Telestroke Versus Nontelestroke. J. Stroke Cerebrovasc. Dis. 2018, 27, 2524-2533. [CrossRef]

11. Wilson, M.E. Stroke: Understanding the differences between males and females. Pflügers Archiv Eur. J. Physiol. 2013, 465, 595-600. [CrossRef]

12. McCullough, L.D.; Alkayed, N.J.; Traystman, R.J.; Williams, M.J.; Hurn, P.D. Postischemic Estrogen Reduces Hypoperfusion and Secondary Ischemia After Experimental Stroke. Stroke 2001, 32, 796-802. [CrossRef]

13. Sun, D.; Tiedt, S.; Yu, B.; Jian, X.; Gottesman, R.F.; Mosley, T.H.; Boerwinkle, E.; Dichgans, M.; Fornage, M. A prospective study of serum metabolites and risk of ischemic stroke. Neurology 2019, 92, e1890-e1898. [CrossRef] [PubMed]

14. Sidorov, E.; Sanghera, D.K.; Vanamala, J.K.P. Biomarker for Ischemic Stroke Using Metabolome: A Clinician Perspective. J. Stroke 2019, 21, 31-41. [CrossRef]

15. Vojinovic, D.; Kalaoja, M.; Trompet, S.; Fischer, K.; Shipley, M.J.; Li, S.; Havulinna, A.S.; Perola, M.; Salomaa, V.; Yang, Q.; et al. Association of Circulating Metabolites in Plasma or Serum and Risk of Stroke. Neurology 2021, 96, e1110-e1123. [CrossRef] [PubMed]

16. Delgado, P.; Chacón, P.; Penalba, A.; Pelegri, D.; García-Berrocoso, T.; Giralt, D.; Santamarina, E.; Ribó, M.; Maisterra, O.; Alvarez-Sabín, J.; et al. Lipoprotein-Associated Phospholipase a 2 Activity Is Associated with Large-Artery Atherosclerotic Etiology and Recurrent Stroke in Tia Patients. Cerebrovasc. Dis. 2012, 33, 150-158. [CrossRef] [PubMed]

17. Thompson, A.; Gao, P.; Orfei, L.; Watson, S.; Di Angelantonio, E.; Kaptoge, S.; Ballantyne, C.M.; Cannon, C.P.; Criqui, M.H.; Cushman, M.; et al. Lipoprotein-associated phospholipase A2 and risk of coronary disease, stroke, and mortality: Collaborative analysis of 32 prospective studies. Lancet 2010, 375, 1536-1544. [CrossRef]

18. Jové, M.; Mauri-Capdevila, G.; Suárez, I.; Cambray, S.; Sanahuja, J.; Quílez, A.; Farré, J.; Benabdelhak, I.; Pamplona, R.; Portero-Otín, M.; et al. Metabolomics predicts stroke recurrence after transient ischemic attack. Neurology 2015, 84, 36-45. [CrossRef]

19. Sun, H.; Kaplan, B.S.; Guan, M.; Zhang, G.; Ye, J.; Long, L.-P.; Blackmon, S.; Yang, C.-K.; Chiang, M.-J.; Xie, H.; et al. Pathogenicity and Transmission of a Swine Influenza a(H6n6) Virus. Emerg. Microbes Infect. 2017, 6, 1-13. [CrossRef]

20. Floegel, A.; Kühn, T.; Sookthai, D.; Johnson, T.; Prehn, C.; Rolle-Kampczyk, U.; Otto, W.; Weikert, C.; Illig, T.; Von Bergen, M.; et al. Serum metabolites and risk of myocardial infarction and ischemic stroke: A targeted metabolomic approach in two German prospective cohorts. Eur. J. Epidemiol. 2018, 33, 55-66. [CrossRef]

21. Harpaz, D.; Bajpai, R.; Ng, G.J.; Soljak, M.; Marks, R.S.; Cheung, C.; Arumugam, T.V.; Quek, A.M.; Tok, A.I.; Seet, R.C. Blood biomarkers to detect new-onset atrial fibrillation and cardioembolism in ischemic stroke patients. Heart Rhythm. 2021, 18, 855-861. [CrossRef] [PubMed]

22. Nathaniel, T.I.; Otukonyong, E.E.; Okon, M.; Chaves, J.; Cochran, T.; Nathaniel, A.I. Metabolic Regulatory Clues from the Naked Mole Rat: Toward Brain Regulatory Functions During Stroke. Brain Res. Bull. 2013, 98, 44-52. [CrossRef] [PubMed]

23. Ormstad, H.; Aass, H.C.D.; Amthor, K.-F.; Lund-Sørensen, N.; Sandvik, L. Serum cytokine and glucose levels as predictors of poststroke fatigue in acute ischemic stroke patients. J. Neurol. 2011, 258, 670-676. [CrossRef]

24. Crimmins, E.M.; Shim, H.; Zhang, Y.S.; Kim, J.K. Differences between Men and Women in Mortality and the Health Dimensions of the Morbidity Process. Clin. Chem. 2019, 65, 135-145. [CrossRef] [PubMed]

25. Redekop, W.K.; Mladsi, D. The Faces of Personalized Medicine: A Framework for Understanding Its Meaning and Scope. Value Health 2013, 16, S4-S9. [CrossRef]

26. Au, A. Metabolomics and Lipidomics of Ischemic Stroke. Int. Rev. Cytol. 2018, 85, 31-69. [CrossRef]

27. Guo, X.; Li, Z.; Zhou, Y.; Yu, S.; Yang, H.; Zheng, L.; Liu, Y.; Sun, Y. Metabolic Profile for Prediction of Ischemic Stroke in Chinese Hypertensive Population. J. Stroke Cerebrovasc. Dis. 2019, 28, 1062-1069. [CrossRef]

28. Colello, M.J.; Ivey, L.E.; Gainey, J.; Faulkner, R.V.; Johnson, A.; Brechtel, L.; Madeline, L.; Nathaniel, T.I. Pharmacological Thrombolysis for Acute Ischemic Stroke Treatment: Gender Differences in Clinical Risk Factors. Adv. Med. Sci. 2018, 63, 100-106. [CrossRef]

29. Lee, Y.; Khan, A.; Hong, S.; Jee, S.H.; Park, Y.H. A metabolomic study on high-risk stroke patients determines low levels of serum lysine metabolites: A retrospective cohort study. Mol. BioSyst. 2017, 13, 1109-1120. [CrossRef]

30. Liu, P.; Li, R.; Antonov, A.A.; Wang, L.; Li, W.; Hua, Y.; Guo, H.; Wang, L.; Liu, P.; Chen, L.; et al. Discovery of Metabolite Biomarkers for Acute Ischemic Stroke Progression. J. Proteome Res. 2017, 16, 773-779. [CrossRef]

31. Tiedt, S.; Brandmaier, S.; Kollmeier, H.; Duering, M.; Artati, A.; Adamski, J.; Klein, M.; Liebig, T.; Holdt, L.M.; Teupser, D.; et al. Circulating Metabolites Differentiate Acute Ischemic Stroke from Stroke Mimics. Ann. Neurol. 2020, 88, 736-746. [CrossRef]

32. Wang, X.; Liu, T.; Song, H.; Cui, S.; Liu, G.; Christoforou, A.; Flaherty, P.; Luo, X.; Wood, L.; Wang, Q.M. Targeted Metabolomic Profiling Reveals Association Between Altered Amino Acids and Poor Functional Recovery After Stroke. Front. Neurol. 2020, 10, 1425. [CrossRef] [PubMed] 
33. Wang, X.; Zhang, L.; Sun, W.; Pei, L.-L.; Tian, M.; Liang, J.; Liu, X.; Zhang, R.; Fang, H.; Wu, J.; et al. Changes of Metabolites in Acute Ischemic Stroke and Its Subtypes. Front. Neurosci. 2021, 14, 1346. [CrossRef] [PubMed]

34. Jickling, G.C.; Sharp, F.R. Blood Biomarkers of Ischemic Stroke. Neurotherapeutics 2011, 8, 349-360. [CrossRef] [PubMed]

35. Weng, H.; Shen, C.; Hirokawa, G.; Ji, X.; Takahashi, R.; Shimada, K.; Kishimoto, C.; Naoharu, I. Plasma Mir-124 as a Biomarker for Cerebral Infarction. Biomed. Res. 2011, 32, 135-141. [CrossRef] [PubMed]

36. Wang, Z.; Lu, G.; Sze, J.; Liu, Y.; Lin, S.; Yao, H.; Zhang, J.; Xie, D.; Liu, Q.; Kung, H.-F.; et al. Plasma miR-124 Is a Promising Candidate Biomarker for Human Intracerebral Hemorrhage Stroke. Mol. Neurobiol. 2018, 55, 5879-5888. [CrossRef]

37. He, X.-W.; Shi, Y.-H.; Liu, Y.-S.; Li, G.-F.; Zhao, R.; Hu, Y.; Lin, C.-C.; Zhuang, M.-T.; Su, J.-J.; Liu, J.-R. Increased Plasma Levels of Mir-124-3p, Mir-125b-5p and Mir-192-5p Are Associated with Outcomes in Acute Ischaemic Stroke Patients Receiving Thrombolysis. J. Atheroscler. 2019, 289, 36-43. [CrossRef] [PubMed]

38. Misra, S.; Kumar, A.; Kumar, P.; Yadav, A.K.; Mohania, D.; Pandit, A.K.; Prasad, K.; Vibha, D. Blood-Based Protein Biomarkers for Stroke Differentiation: A Systematic Review. J. Proteomics 2017, 11, 1700007. [CrossRef]

39. Farooqi, I.; Collet, T.; Sonoyama, T.; Henning, E.; Keogh, J.; Ingram, B.; Kelway, S.; Guo, L. A Metabolomic Signature of Acute Caloric Restriction. J. Clin. Endocrinol. Metab. 2018, 102, 4486-4495. [CrossRef]

40. Stettin, D.; Poulin, R.X.; Pohnert, G. Metabolomics Benefits from Orbitrap Gc-Ms-Comparison of Low- and High-Resolution Gc-Ms. Metabolites 2020, 10, 4. [CrossRef]

41. DeHaven, C.D.; Evans, A.M.; Dai, H.; A Lawton, K. Organization of GC/MS and LC/MS metabolomics data into chemical libraries. J. Cheminformatics 2010, 2, 9. [CrossRef]

42. Kaare, P.D.; Andersen, G.; Ingeman, A.; Krog, B.R.; Bartels, P.; Søren, P. Sex-Related Differences in Quality of Care and Short-Term Mortality among Patients with Acute Stroke in Denmark: A Nationwide Follow-up Study. J. Stroke 2009, 40, 1134-1139.

43. George, M.G.; Tong, X.; Kuklina, E.V.; Darwin, R. Trends in Stroke Hospitalizations and Associated Risk Factors among Children and Young Adults, 1995-2008. J. Ann. Neurol. 2011, 70, 713-721. [CrossRef] [PubMed]

44. Barber, P.A.; Demchuk, A.M.; Zhang, J.; Buchan, A.M. Validity and reliability of a quantitative computed tomography score in predicting outcome of hyperacute stroke before thrombolytic therapy. Lancet 2000, 355, 1670-1674. [CrossRef]

45. Kamtchum-Tatuene, J.; Jickling, G.C. Blood Biomarkers for Stroke Diagnosis and Management. NeuroMolecular Med. 2019, 21, 344-368. [CrossRef]

46. Johnson, C.; Ivanisevic, J.; Siuzdak, G. Metabolomics: Beyond biomarkers and towards mechanisms. Nat. Rev. Mol. Cell Biol. 2016, 17, 451-459. [CrossRef]

47. Tate, M.J.; Shugart, R.M.; Moraney, R.A.; Bechtel, L.E.; Blum, B.; Nathaniel, I.T. Gender and functional outcomes in pre-stroke depressive patients treated with thrombolytic therapy. Future Neurol. 2020, 15, 28-35. [CrossRef]

48. Rotimi, O.R.; Ajani, I.F.; Penwell, A.; Lari, S.; Walker, B.; I Nathaniel, T. In acute ischemic stroke patients with smoking incidence, are more women than men more likely to be included or excluded from thrombolysis therapy? Women's Health 2020, 16, 1745506520922760. [CrossRef]

49. Syme, C.; Czajkowski, S.; Shin, J.; Abrahamowicz, M.; Leonard, G.; Perron, M.; Richer, L.; Veillette, S.; Gaudet, D.; Strug, L.; et al. Glycerophosphocholine Metabolites and Cardiovascular Disease Risk Factors in Adolescents: A Cohort Study. Circulation 2016, 134, 1629-1636. [CrossRef] [PubMed]

50. Marathe, G.K.; Pandit, C.; Lakshmikanth, C.L.; Chaithra, V.H.; Jacob, S.P.; D'Souza, C.J.M. To hydrolyze or not to hydrolyze: The dilemma of platelet-activating factor acetylhydrolase. J. Lipid Res. 2014, 55, 1847-1854. [CrossRef]

51. Stegemann, C.; Pechlaner, R.; Willeit, P.; Langley, S.R.; Mangino, M.; Mayr, U.; Menni, C.; Moayyeri, A.; Santer, P.; Rungger, G.; et al. Lipidomics Profiling and Risk of Cardiovascular Disease in the Prospective Population-Based Bruneck Study. Circulation 2014, 129, 1821-1831. [CrossRef] [PubMed]

52. Cheng, M.; Bhujwalla, Z.M.; Glunde, K. Targeting Phospholipid Metabolism in Cancer. Front. Oncol. 2016, 6, 266. [CrossRef] [PubMed]

53. Van Der Kemp, W.J.M.; Stehouwer, B.L.; Runge, J.H.; Wijnen, J.P.; Nederveen, A.J.; Luijten, P.R.; Klomp, D.W.J. Glycerophosphocholine and Glycerophosphoethanolamine Are Not the Main Sources of the In Vivo31P MRS Phosphodiester Signals from Healthy Fibroglandular Breast Tissue at 7 T. Front. Oncol. 2016, 6, 29. [CrossRef]

54. Wigle, T.J.; Tsvetkova, E.V.; Welch, S.A.; Kim, R.B. DPYD and Fluorouracil-Based Chemotherapy: Mini Review and Case Report. Pharmaceutics 2019, 11, 199. [CrossRef]

55. Detailleur, S.; Segelov, E.; Re, M.D.; Prenen, H. Dihydropyrimidine Dehydrogenase Deficiency in Patients with Severe Toxicity after 5-Fluorouracil: A Retrospective Single-Center Study. Ann. Gastroenterol. 2021, 34, 68-72.

56. Loriot, M.A.; Ciccolini, J.; Thomas, F.; Barin-Le-Guellec, C.; Royer, B.; Milano, G.; Picard, N.; Becquemont, L.; Verstuyft, C.; Narjoz, C.; et al. Dihydropyrimidine Déhydrogenase (Dpd) Deficiency Screening and Securing of Fluoropyrimidine-Based Chemotherapies: Update and Recommendations of the French Gpco-Unicancer and Rnpgx Networks. Bull. Cancer 2018, 105, 397-407. [CrossRef]

57. Kristensen, M.; Pedersen, P.; Mejer, J. The Value of Dihydrouracil/Uracil Plasma Ratios in Predicting 5-Fluorouracil-Related Toxicity in Colorectal Cancer Patients. J. Int. Med. Res. 2010, 38, 1313-1323. [CrossRef]

58. Sistonen, J.; Büchel, B.; Froehlich, T.K.; Kummer, D.; Fontana, S.; Joerger, M.; van Kuilenburg, A.B.; Largiadèr, C.R. Predicting 5-fluorouracil toxicity: DPD genotype and 5,6-dihydrouracil:uracil ratio. Pharmacogenomics 2014, 15, 1653-1666. [CrossRef] 
59. Basbous, J.; Aze, A.; Chaloin, L.; Lebdy, R.; Hodroj, D.; Ribeyre, C.; Larroque, M.; Shepard, C.; Kim, B.; Pruvost, A.; et al. Dihydropyrimidinase Protects from DNA Replication Stress Caused by Cytotoxic Metabolites. Immunol. Cancer Biol. 2021, 48, 1886-1904. [CrossRef] [PubMed]

60. Handa, R.J.; Sharma, D.; Uht, R. A Role for the Androgen Metabolite, 5alpha Androstane 3beta, 17beta Diol (3ß-Diol) in the Regulation of the Hypothalamo-Pituitary-Adrenal Axis. Front. Endocrinol. (Lausanne) 2011, 2, 65. [CrossRef]

61. Handa, R.J.; Weiser, M.J.; Zuloaga, D.G. A Role for the Androgen Metabolite, 5alpha-Androstane-3beta,17beta-Diol, in Modulating Oestrogen Receptor Beta-Mediated Regulation of Hormonal Stress Reactivity. J. Neuroendocrinol. 2009, 21, 351-358. [CrossRef]

62. Mero, A.A.; Ojala, T.; Hulmi, J.J.; Puurtinen, R.; Karila, T.A.; Seppälä, T. Effects of alfa-hydroxy-isocaproic acid on body composition, DOMS and performance in athletes. J. Int. Soc. Sports Nutr. 2010, 7, 1. [CrossRef]

63. Ojala, T.; Wilson, J.M.; Hulmi, J.J.; Karila, T.; Seppälä, T.A.; Mero, A.A. Chapter 21—A-Hydroxy-Isocaproic Acid (Hica)—Effects on Body Composition, Muscle Soreness and Athletic Performance. In Nutrition and Enhanced Sports Performance; Bagchi, D., Nair, S., Sen, C.K., Eds.; Academic Press: San Diego, CA, USA, 2013; pp. 213-216.

64. Sakko, M.; Tjäderhane, L.; Sorsa, T.; Hietala, P.; Järvinen, A.; Bowyer, P.; Rautemaa, R. 2-Hydroxyisocaproic acid (HICA): A new potential topical antibacterial agent. Int. J. Antimicrob. Agents 2012, 39, 539-540. [CrossRef]

65. Kuhara, T.; Shinka, T.; Inoue, Y.; Matsumoto, M.; Yoshino, M.; Sakaguchi, Y.; Matsumoto, I. Studies of Urinary Organic Acid Profiles of a Patient with Dihydrolipoyl Dehydrogenase Deficiency. Clin. Chim. Acta 1983, 133, 133-140.

66. Sumi, K.; Sakuda, M.; Munakata, K.; Nakamura, K.; Ashida, K. $\alpha$-Hydroxyisocaproic Acid Decreases Protein Synthesis but Attenuates TNF $\alpha /$ IFN $\gamma$ Co-Exposure-Induced Protein Degradation and Myotube Atrophy via Suppression of iNOS and IL-6 in Murine C2C12 Myotube. Nutrients 2021, 13, 2391. [CrossRef]

67. Nathaniel, T.I.; Stewart, B.; Williams, J.; Hood, M.; Imeh-Nathaniel, A. A New Insight into the Ability to Resist Ischemic Brain Injury: Does Hibernation Matter?: An Editorial Comment for 'Arctic Ground Squirrel Hippocampus Tolerates Oxygen Glucose Deprivation Independent of Hibernation Season Even When Not Hibernating and after Atp Depletion, Acidosis and Glutamate Efflux'. J. Neurochem. 2017, 142, 10-13. [PubMed]

68. Salminen, A.; Hyttinen, J.M.; Kaarniranta, K. Amp-Activated Protein Kinase Inhibits Nf-Kb Signaling and Inflammation: Impact on Healthspan and Lifespan. J. Mol. Med. (Berlin) 2011, 89, 667-676. [CrossRef] [PubMed]

69. Ebner, N.; Sliziuk, V.; Scherbakov, N.; Sandek, A. Muscle wasting in ageing and chronic illness. ESC Hear. Fail. 2015, 2, 58-68. [CrossRef]

70. Zhao, C.; Huang, H.; Pan, Q.; Huang, W.; Peng, W.; Xu, H.; Feng, Z.; Du, Y.; Nie, Y.; Zhou, Y. Unconjugated Bilirubin Attenuates DSS-Induced Colitis Potentially via Enhancement of Bilirubin Reabsorption. Front. Pharmacol. 2021, 12. [CrossRef]

71. Liu, Y.; Li, P.; Lu, J.; Xiong, W.; Oger, J.; Tetzlaff, W.; Cynader, M. Bilirubin Possesses Powerful Immunomodulatory Activity and Suppresses Experimental Autoimmune Encephalomyelitis. J. Immunol. 2008, 181, 1887-1897. [CrossRef] [PubMed]

72. Allen, C.L.; Bayraktutan, U. Oxidative Stress and Its Role in the Pathogenesis of Ischaemic Stroke. Int. J. Stroke 2009, 4, 461-470. [CrossRef] [PubMed]

73. Dohi, K.; Mochizuki, Y.; Satoh, K.; Jimbo, H.; Hayashi, M.; Toyoda, I.; Ikeda, Y.; Abe, T.; Aruga, T. Transient elevation of serum bilirubin (a heme oxygenase-1 metabolite) level in hemorrhagic stroke: Bilirubin is a marker of oxidant stress. Brain Edema XII 2003, 86, 247-249. [CrossRef]

74. Ohira, T.; Peacock, J.M.; Iso, H.; Chambless, L.E.; Rosamond, W.D.; Folsom, A.R. Serum and Dietary Magnesium and Risk of Ischemic Stroke: The Atherosclerosis Risk in Communities Study. Am. J. Epidemiol. 2009, 169, 1437-1444. [CrossRef] [PubMed]

75. Zhao, B.; Hu, L.; Dong, Y.; Xu, J.; Wei, Y.; Yu, D.; Xu, J.; Zhang, W. The Effect of Magnesium Intake on Stroke Incidence: A Systematic Review and Meta-Analysis with Trial Sequential Analysis. Front. Neurol. 2019, 10, 852. [CrossRef]

76. Fleming, T.; Blum, B.; Averkamp, B.; Sullivan, J.; Nathaniel, T. Effect of Antihypertensive Medications on Thrombolysis Therapy and Outcomes in Acute Ischemic Stroke Patients. J. Clin. Hypertens. 2019, 21, 271-279. [CrossRef] [PubMed] 